Introduction/Background* An increased risk of endometrial cancer and other endometrial pathologies is associated with the use of tamoxifen. Our objective is to evaluate the hysteroscopic approach on patients who had received tamoxifen as an adjuvant therapy for a breast cancer.

Methodology Retrospective observational study of 7406 office hysteroscopies performed between January 2010 and December 2019 at our university hospital. 213 patients who have received tamoxifen were found and included on this study.

Result(s)* The mean age was 53.6 \pm 10.1 years. 167 patients (78\%) were postmenopausal, while 45 (22\%) were premenopausal.

138 office hysteroscopies were performed on symptomatic patients: 128 patients (60\%) with postmenopausal bleeding and 10 patients (5\%) with hypermenorrhea. Besides, 66 asymptomatic patients (30\%) with abnormal ultrasound underwent an office hysteroscopy.

Ultrasound performed before hysteroscopy allowed to reveal 86 endometrial polyps (41\%), 10 submucosal myomas $(5 \%)$ and 11 patients with endometrial increased thickness (5\%). On the 105 patients left (50\%), ultrasound was normal.

17 office hysteroscopies could not be performed because of cervical stenosis. We found 88 (42\%) endometrial polyps, 7 (3\%) submucosal myomas and $3(2 \%)$ uterine malformations. $85(41 \%)$ patients underwent the hysteroscopy without abnormal findings. Hysteroscopy was suggestive of endometrial hyperplasia on 8 patients (4\%). Findings were highly suggestive of endometrial carcinoma on 4 patients $(2 \%)$.

On the histologic examination, malignancy was confirmed on the 4 patients with hysteroscopy suggestive of endometrial adenocarcinoma, while endometrial hyperplasia was confirmed on 13 patients (6\%).

Two of the four patients with diagnosis of endometrial adenocarcinoma were symptomatic (postmenopausal bleeding). Ultrasound on these patients showed endometrial polyps. The other two patients were performed a hysteroscopy because of increased endometrial thickness. Hysteroscopy revealed endometrial neoformations with atypical vascularization.

Conclusion* Our results show the increased risk of endometrial pathology on tamoxifen-treated patients, according to the existing literature. Ultrasound can be a good first approach on asymptomatic patients, but with limited accuracy. However, hysteroscopy is the best way of evaluating the endometrial cavity, making possible to see and treat endometrial pathologies on the same procedure. Furthermore, more studies are needed to validate the use of hysteroscopy as a first approach on symptomatic patients.

\section{PAZODOBLE - PAZOPANIB VS. PAZOPANIB PLUS GEMCITABINE IN RELAPSED OR METASTATIC UTERINE LEIOMYOSARCOMAS OR UTERINE CARCINOSACOMAS}

${ }^{1}$ LA Otten*, ${ }^{2}$ S Sehouli, ${ }^{3} E M$ Grischke, ${ }^{4} D$ Pink, ${ }^{5} P$ Reichardt, ${ }^{6} \mathrm{P}$ Harter, ${ }^{7} \mathrm{P}$ Wimberger, ${ }^{8} \mathrm{Z}$ Alwafai, ${ }^{9} \mathrm{BB}$ Runnebaum, ${ }^{1} \mathrm{~A}$ Mustea. ${ }^{1}$ Universitätsklinikum Bonn, Klinik für Gynäkologie und Gynäkologische Onkologie; ${ }^{2}$ Universitätsmedizin Berlin Charité Campus VirchowKlinikum; ${ }^{3}$ Universitätsfrauenklinik Tübingen; ${ }^{4}$ Helios Klinikum Bad Saarow, Hämatologie, Onkologie und Palliativmedizin, Sarkomzentrum; ${ }^{5}$ Helios Klinikum Berlin-Buch, Klinik für Onkologie und Paliativmedizin; ${ }^{6}$ Ev. KlinikenEssen-Mitte, Department of Gynecology and Gynecologic Oncology; ' Universitätsklinik Carl Gustav Carus der Technischen Universität Dresden, Klinik für Frauenheilkunde und Geburtshilfe; ${ }^{8}$ Universitätsmedizin Greifswald, Klinik und Poliklinik für Frauenheilkunde und Geburtshilfe; ${ }^{9}$ Universitätsklinikum Jena

\subsection{6/ijgc-2021-ESG0.253}

Introduction/Background* As there are only a few therapy options for advanced or recurrent leiomyosarcoma (LMS) and Carcinosarcomas (CS), new effective drug combinations with an acceptable toxicity profile are urgently needed.

Pazopanib is an orally available second-generation multi targeted tyrosine kinase inhibitor (TKI), targeting among others VEGFR, PFGG and c-kitand is approved in soft tissue sarcomas.

A phase I study evaluated the combination of pazopanib and gemcitabine and showed an acceptable toxicity profile. We aim to test the activity of the combination therapy in patients with LMS in a randomized setting and to describe the activity in CS.

Methodology The PazoDoble study is a prospective, randomized, open-label, multicentre phase II trial. Patients with relapsed or metastatic uterine LMS or uterine CS are eligible for participation. LMS are randomised in a $1: 1$ fashion into two treatment arms. A: pazopanib $800 \mathrm{mg}$ p.o./d plus gemcitabine $1000 \mathrm{mg} / \mathrm{m} 2$ i.v. over $30 \mathrm{~min} \mathrm{~d} 1$ und $\mathrm{d} 8 \mathrm{q} 3 \mathrm{w}$ or $\mathrm{B}$ : pazopanib $800 \mathrm{mg}$ p.o./d. Uterine CS will be enrolled only in Arm A. Duration of therapy will be maximum 18 months or until progression, death or unacceptable toxicity. Primary Endpoints are the six month PFS and clinical progression; Secondary endpoints are the objective response rate, duration of response, time to progression, OS, toxicity and tolerability and quality of life. A previousatment with doxorubicin or any contraindications against doxorubicin and an ECOG of 0 or 1 are mandatory. Clinical and imaging follow-up are scheduled every three month until disease progression or death.

Enrolment was started in October 2019 at nine sites. Until now, 12 Patients are enrolled (5 CS and 7 LMS). We aim to include 87 patients with LMS and 20 with CS. The primary endpoint will be analysed as the ratio of the number of patients showing no progress after 6 month divided by the
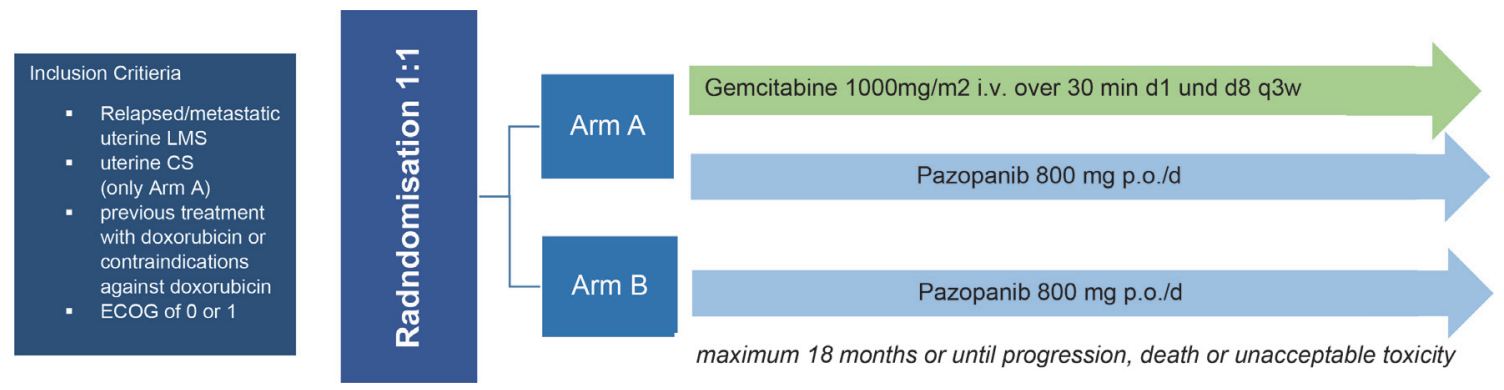

Abstract 169 Figure 1 
number of evaluable patients. A drop-out rate of 5\% was assumed.

Result(s)* Conclusion* In the clinical setting of advanced and recurrent LMS and CS there are no well-evaluated therapies available. This trial is clinically highly relevant and offers opportunity for patients to receive promising therapy.

\section{HIV TESTING IN CERVICAL DYSPLASIA, PRACTITIONERS' OPINION}

${ }^{1} \mathrm{R}$ Van de Laar*, ${ }^{2} \mathrm{C}$ Jordans, ${ }^{3} \mathrm{C}$ Rokx, ${ }^{1} \mathrm{HJ}$ Van Beekhuizen, ${ }^{1} \mathrm{HC}$ Van Doorn. ${ }^{1}$ Erasmus University Medical Center, Gynaecologic oncology, Rotterdam, Netherlands; ${ }^{2}$ Erasmus University Medical Center, Microbiology and Infectious Diseases, Rotterdam, Netherlands; ${ }^{3}$ Erasmus University Medical Center, Internal medicine, section Infectious Diseases, Rotterdam, Netherlands

\subsection{6/ijgc-2021-ESG0.254}

Introduction/Background* Cervical dysplasia is an HIV indicator condition and according international recommendations HIV testing is strongly advised in women with cervical dysplasia, because the risk of an undiagnosed HIV is thought to be $>0.1 \%$. Therefore an HIV test should be offered to all women with cervical dysplasia. There is no literature about the opinion of Gynaecologist on HIV screening in patients with cervical dysplasia.

Methodology We sent an online questionnaire to gynecologist in South West Netherlands to investigate 1) what they know about this issue, 2) their opinion and willingness on active HIV testing for this cervical dysplasia.

Result(s)* The questionaire was sent to 103 gynaecologists of whom fifty-six participants replied (54\%). Forty-eight (86\%) think patients are not offended when HIV testing is offered and $50(89 \%)$ have no difficulty to address HIV testing. Thirty-nine (70\%) gynaecologist think that the prevalence of undiagnosed HIV infection is lower than $0.1 \%$, and only seven $(12,5 \%)$ accept HIV testing in case of a prevalence of $0.1 \%$ or less. Thirthy-two $(57 \%)$ are willing to test with a prevalence of $1 \%$ or higher.

Conclusion* To address and offer HIV testing seems not an issue for the gynaecologists questioned in our study. However, the willingness to routinely perform an HIV test for cervical dysplasia at the assumed $0.1 \%$ prevalence looks insufficient and differs from the recommendations of international policy makers. Discussion is needed tot change the treshold or the willingness for testing.

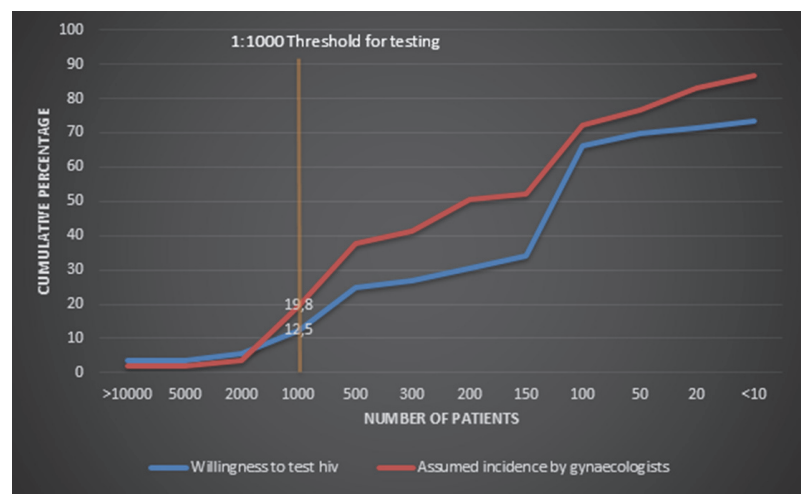

Abstract 206 Figure 1

\section{2 \\ EVALUATION OF UPA/PAI-1 AS A PROGNOSTIC MARKER IN YOUNG WOMEN WITH BREAST CANCER}

${ }^{1 ; 2} \mathrm{M}$ Sobočan*, ${ }^{2} \mathrm{U}$ Dimnik, ${ }^{2} \mathrm{~B}$ Dežman, ${ }^{2} \mathrm{M}$ Turk, ${ }^{3} \mathrm{R}$ Kavalar, ${ }^{1 ; 2}$ I Takač, ${ }^{1 ; 2} \mathrm{D}$ Arko. ${ }^{1}$ University Medical Centre Maribor, Division for Gynaecology and Perinatology, Maribor, Slovenia; ${ }^{2}$ University of Maribor, Faculty of Medicine, Maribor, Slovenia; ${ }^{3}$ University Medical Centre Maribor, Department of Pathology, Maribor, Slovenia

\subsection{6/ijgc-2021-ESG0.255}

Introduction/Background* Breast cancer is uncommon in young women in developed countries. It is very heterogenous disease and studies showed, that young patients often have biologically complex and often more agressive tumours. However, little is known on markers available to improve assessment of prognosis or provide an additional therapeutic targets. One potential marker is the uPA-PAI-1 complex or each of the proteins individually. The protease uPA and its inhibitor PAI-1 have been implicated in cancer progression through facilitating tumour cell migration. The aim of this study was to evaluate the potential impact of uPA and PAI-1 as prognostic markers in young women with breast cancer.

Methodology We identified through the use of our institutional database on breast cancer 84 from 2283 (3.7\%) of women diagnosed with breast cancer under the age of 45 years at the University Medical Centre Maribor, Slovenia between January 2009 - December 2019. An exam of clinical patient records was performed and clinico-pathological data were evaluated. Tumour tissue was prospectively analysed after primary surgical treatment and quantified using immunometric method ELISA sets. Values of uPA and PAI-1 were expressed in $\mathrm{ng} / \mathrm{mg}$ of proteins. Correlations were evaluated using the Spearman rank test and continous data were compared using the Mann-Whitney U test. Data were evaluated using the SPSS for Mac version 23.0

Result(s)* Clinical data were available for 70 women with BC. Data on uPA/PAI-1 protein levels and the expression of the uPA-PAI-1 complex were available in 39 women (55.7\%). The median age of patients in our study was 42.0 years (29-45). The complex of uPA/PAI-1 was significantly correlated with age at time of diagnosis $\left(r_{s}=-.366\right.$, $\mathrm{p}<0.022)$. There was no significant correlation between the expression levels of uPA and levels of PAI-1 and oestrogen receptors, progesterone receptors, Ki-67 expression or tumour size. uPA/PAI-1 complex was also not significantly correlated with the hormone receptor negative (TNBC) breast cancer $(\mathrm{p}>.814)$.

Conclusion* Further research should evaluate the connection of age and the uPA/PAI-1 complex. uPA and PAI-1 did not show independant prognostic abilities in this pilot study.

\section{INCIDENCE OF GYNAECOLOGICAL CANCER DURING THE COVID-19 PANDEMIC}

${ }^{1} \mathrm{E}$ Oymans ${ }^{*},{ }^{2} \mathrm{C}$ De Kroon, ${ }^{3} \mathrm{~J}$ Bart, ${ }^{4} \mathrm{M}$ Van der $\mathrm{Aa},{ }^{5} \mathrm{HW}$ Nijman. ${ }^{1} / \mathrm{KNL}$, Utrecht, Netherlands; ${ }^{2}$ Leiden University Medical Center (LUMC), Gynaecological oncology, Leiden, Netherlands; ${ }^{3}$ University Medical Center Groningen (UMCG), Pathology, Groningen, Netherlands; ${ }^{4}$ KNNL, Enschede, Netherlands; ${ }^{5}$ University of Groningen, University Medical Center Groningen, Department of Obstetrics and Gynecology, The Netherlands

10.1136/ijgc-2021-ESG0.256 